\title{
Preparation of poly(ethylene glycol)/polylactide hybrid fibrous scaffolds for bone tissue engineering
}

This article was published in the following Dove Press journal:

International Journal of Nanomedicine

29 November 201I

Number of times this article has been viewed

PeiYan Ni

ShaoZhi Fu

Min Fan

Gang Guo

Shuai Shi

JinRong Peng

Feng Luo

ZhiYong Qian

State Key Laboratory of Biotherapy and Cancer Center, West China

Hospital, West China Medical School, Sichuan University, Chengdu, Sichuan, People's Republic of China
Correspondence: ZhiYong Qian State Key Laboratory of Biotherapy and Cancer Center, West China Hospital, West China Medical School, Sichuan University, Chengdu, Sichuan 61004I, People's Republic of China Tel +862885164063

Email anderson-qian@163.com
Abstract: Polylactide (PLA) electrospun fibers have been reported as a scaffold for bone tissue engineering application, however, the great hydrophobicity limits its broad application. In this study, the hybrid amphiphilic poly(ethylene glycol) (PEG)/hydrophobic PLA fibrous scaffolds exhibited improved morphology with regular and continuous fibers compared to corresponding blank PLA fiber mats. The prepared PEG/PLA fibrous scaffolds favored mesenchymal stem cell (MSC) attachment and proliferation by providing an interconnected porous extracellular environment. Meanwhile, MSCs can penetrate into the fibrous scaffold through the interstitial pores and integrate well with the surrounding fibers, which is very important for favorable application in tissue engineering. More importantly, the electrospun hybrid PEG/PLA fibrous scaffolds can enhance MSCs to differentiate into bone-associated cells by comprehensively evaluating the representative markers of the osteogenic procedure with messenger ribonucleic acid quantitation and protein analysis. MSCs on the PEG/PLA fibrous scaffolds presented better differentiation potential with higher messenger ribonucleic acid expression of the earliest osteogenic marker Cbfa- 1 and mid-stage osteogenic marker Col I. The significantly higher alkaline phosphatase activity of the PEG/PLA fibrous scaffolds indicated that these can enhance the differentiation of MSCs into osteoblast-like cells. Furthermore, the higher messenger ribonucleic acid level of the late osteogenic differentiation markers OCN (osteocalcin) and OPN (osteopontin), accompanied by the positive Alizarin red S staining, showed better maturation of osteogenic induction on the PEG/PLA fibrous scaffolds at the mineralization stage of differentiation. After transplantation into the thigh muscle pouches of rats, and evaluating the inflammatory cells surrounding the scaffolds and the physiological characteristics of the surrounding tissues, the PEG/PLA scaffolds presented good biocompatibility. Based on the good cellular response and excellent osteogenic potential in vitro, as well as the biocompatibility with the surrounding tissues in vivo, the electrospun PEG/PLA fibrous scaffolds could be one of the most promising candidates in bone tissue engineering.

Keywords: electrospinning, fibrous scaffolds, poly(ethylene glycol)/polylactide, mesenchymal stem cells, bone tissue engineering

\section{Introduction}

Bone defects resulted from trauma, abnormalities, infection, or tumor resection have presented a severe challenge for the regeneration of cartilage, bone, and teeth in the tissue regeneration field. As the traditional treatments for bone lesions, autologous bone grafts are restricted because of donor site morbidity and potential secondary wounds, while allogeneic bone grafts are limited because of the risk of potential transmission of pathogens. ${ }^{1}$ As a consequence, various bone substitutes with similar structure and function to autologous bones have been proposed. As temporary templates for cell 
seeding, migration, proliferation, and differentiation prior to the regeneration of natural extracellular matrix (ECM) or biological functional tissue, ${ }^{2,3}$ biomaterial scaffolds for bone tissue engineering applications require consideration of proper toughness for cell adhesion, porous three-dimensional structure for cell migration and new bone tissue ingrowth, as well as good osteoconductivity to promote bone repair. ${ }^{4,5}$ In recent years, electrospun fibers as bone scaffolds have received great attention for the following reasons: (1) their morphologies are similar to the structures of natural ECM, (2) their intrinsic large surface area-to-volume ratios offer sufficient space for cell adhesion and proliferation, and (3) their interconnected porous structures can provide windows for nutrient and metabolic waste exchange, ${ }^{6,7}$ as well as promote vascularization to apply nutrient and drain the consumed metabolites in vivo. ${ }^{8}$

The electrospinning technique was originally developed by Zeleny in $1914,{ }^{9}$ and was first patented in the 1930 s. $^{10}$ Electrospinning is a remarkably simple and versatile technique capable of producing microscale or nanoscale polymer fibers directly from a variety of polymers and composite materials. However, there was little interest in electrospinning or electrospun fibers outside of the filter industry until the mid-1990s. ${ }^{11}$ As a remarkably simple and versatile technique, electrospinning has received great attention in a broad range of biomedical applications, such as sensors, ${ }^{12}$ photovoltaic cells, ${ }^{13}$ chemically- and biologically-resistant clothing, carriers for drug delivery system, barriers for the prevention of adhesion, substrates for wound dressing, supports for biocatalysis, active components for biosensing, ${ }^{14}$ and especially as scaffolds for tissue engineering during the past two decades. To be successfully applied in bone tissue engineering, electrospun fibers essentially need to have good cell responses and osteoconductivity. In fact, electrospun nonwoven polymer fabrics have been extensively applied as scaffolds for bone-like cells ${ }^{15-17}$ and mesenchymal stem cells (MSCs) $)^{18,19}$ based on the advantages of operation facility, easy accommodation, and interconnected porous structures. Furthermore, some studies have certified that electrospun fibers alone can be tailored to fabricate functional scaffolds in bone tissue engineering and enhance bone regeneration to a certain extent without any inflammatory reaction. ${ }^{11,20}$

During recent decades, many electrospun polymers have been used for bone tissue engineering, which include both nonbiodegradable and biodegradable polymers either with a synthetic or natural origin. Among them, nonbiodegradable electrospun polymers such as polyurethane and polyester urethane could interfere with tissue turnover and remodeling due to their slow degradation, even though they possessed substantial mechanical stability. ${ }^{21,22}$ Many biodegradable electrospun polymers have been fabricated such as collagen, ${ }^{23}$ chitosan, ${ }^{24}$ poly(glycolic acid), ${ }^{25}$ polylactide (PLA), ${ }^{26}$ poly(E-caprolactone), ${ }^{27}$ poly(lactide-co-glycolide), ${ }^{28}$ and polyanhydride. ${ }^{29}$ Among the biodegradable electrospun polymers, PLA has been used clinically in bone repair due to its tunable mechanical properties, and it can degrade gradually to natural metabolites along with the construction of new tissue and finally be replaced by the new tissue. However, based on their inherent hydrophobic nature, the resorbable biocompatible PLA scaffolds may reduce expression of phenotypic markers by adherent osteoblasts, ${ }^{30}$ and further influence cell attachment and proliferation due to the denaturation of adhesive ligands of cells, ${ }^{24,31}$ which are far from desirable. During recent years, some efforts have been undertaken to graft hydrophilic moieties in order to make PLA scaffolds more conducive for cell attachment, proliferation, and differentiation. As reported, the blending of amphiphilic polyether poly(ethylene glycol) (PEG) to PLA can modulate the inherent hydrophilicity property, crystallinity, tensile strength, and degradation behaviors. ${ }^{32}$ Accordingly, electrospun hybrid scaffolds were prepared in this study by blending PLA with amphiphilic PEG as filler to tailor the scaffolds' hydrophilic properties, and to improve cell responses such as cell attachment, proliferation, and osteoblastic differentiation. . $^{3,34}$

MSCs isolated from bone marrow are multipotent cells that can be induced to differentiate into a variety of mesenchymal tissues including bone, cartilage, tendon, fat, and muscle both in vitro and in vivo. ${ }^{1}$ MSCs have been widely applied in tissue engineering because they are easy to get and manipulate, can be easily differentiated, are biocompatible almost without immune response or tumorigenesis, and especially because they pose no ethical or legal problems. ${ }^{35}$ Therefore, MSCs are well suited to act as seed cells for successful tissue renewal and replacement of damaged and diseased musculoskeletal tissues in bone tissue engineering. ${ }^{36}$

In this study, the uniform and continuous PEG/PLA fibrous scaffolds presented good cell responses and excellent osteogenic ability in the MSCs differentiation procedure. For further application in bone repair in vivo, the biocompatibility of the fibrous scaffolds were specially examined with hematoxylin and eosin staining after being transplanted into the thigh muscle pouches of rats. All the results demonstrated that the PEG/PLA hybrid fibrous scaffolds showed ideal potential for broad application in bone tissue engineering. 


\section{Materials and methods Materials}

Semicrystalline grade PLA 4032D with approximately 2\% of D-lactic acid monomer used in this study was supplied by NatureWorks LLC (Minneapolis, MN). PEG (number average molecular weight $=4000$ ) was purchased from Sigma-Aldrich (St Louis, MO). PLA and PEG were dried in a vacuum oven at $110^{\circ} \mathrm{C}$ and $50^{\circ} \mathrm{C}$ for 24 hours before use, respectively. Dichloromethane was obtained from Kelong Chemical (Chengdu, China) and used as solvent. All other used chemical agents were of analytical reagent grade.

\section{Electrospinning process}

A known amount of dried PEG and PLA (PEG/PLA = 5/95) were dissolved in dichloromethane with vigorous stirring for 6 hours to form a $60 \mathrm{mg} / \mathrm{mL}$ transparent and homogeneous solution. PEG/PLA solution was electrospun at a high voltage of $18 \mathrm{kV}$ driven by a high voltage power supply (High Voltage Technology Institute, Beijing, China) under $60 \%$ humidity at room temperature. The obtained solution was directly transferred into a $20 \mathrm{~mL}$ plastic syringe (Becton, Dickinson and Company, East Rutherford, NJ) at the feeding rate of $6 \mathrm{~mL} /$ hour, which was set by a syringe pump (Smiths Medical Instrument Company, Zhejiang, China). The spinneret was capped with a blunt-end needle with an inner diameter of $0.5 \mathrm{~mm}$, and the distance between the spinneret and the grounded aluminum foil collector was $12 \mathrm{~cm}$. Pure PLA fibers were also prepared in the same processing conditions for comparison. All obtained electrospun fibrous scaffolds were dried under vacuum for 48 hours to eliminate any potential residual solvents and kept in a desiccator for further characterization and application.

\section{Morphological characterization}

In order to investigate the surface morphologies and the microstructure of the electrospun PEG/PLA hybrid fibrous scaffold, scanning electron microscopy (SEM) analysis was conducted (JSM-5900 Low Vacuum SEM; JEOL, Tokyo, Japan). After being seeded with cells for predetermined time, the fibrous scaffolds were fixed using $2.5 \%$ glutaraldehyde overnight at $4^{\circ} \mathrm{C}$. Subsequently, they were dehydrated in ethanol with a series of concentrations ( $30 \%, 50 \%, 70 \%, 80 \%, 90 \%, 95 \%$, and $100 \%$ ) and dried in vacuum. All the samples were sputter-coated with a thin layer of gold using an SBC-12 Ion Sputtering Coater (KYKY Technology Development Ltd, Beijing, China) and observed at $20 \mathrm{kV}$ accelerating voltage. The average diameters of the electrospun fibers were obtained by analyzing at least 40 measurements randomly per specimen.

\section{Isolation and culture of rat bone marrow MSCs}

The neonate Sprague Dawley rats (3 days old) were purchased from the Laboratory Animal Center of Sichuan University (Chengdu, China). The experiments were performed based on the Principles of Laboratory Animal Care formulated by the National Society for Medical Research and the Guide for the Care and Use of Laboratory Animals, ${ }^{37}$ and all protocols were operated according to the Animal Care and Use Committee of West China Hospital, Sichuan University. The fresh femur were harvested aseptically from the neonate rats which were anesthetized and sterilized with alcohol and povidine. ${ }^{38}$ The bone marrow was flushed out with Dulbecco's modified Eagle's medium (Life Technologies, Carlsbad, CA) supplemented with 10\% fetal bovine serum (Thermo Fisher Scientific, Waltham, MA), 100 unit $/ \mathrm{mL}$ penicillin (Life Technologies) and $100 \mu \mathrm{g} / \mathrm{mL}$ streptomycin (Life Technologies) using a $1 \mathrm{~mL}$ syringe. The obtained solution was cultured in a $75 \mathrm{~cm}^{2}$ tissue culture flask at $37^{\circ} \mathrm{C}$, $5 \%$ carbon dioxide. Culture medium was changed every 3-4 days, and MSCs approached confluency after about 1 week. Rat bone MSCs were trypsinized briefly with $0.25 \%$ trypsin and subcultured at 1:3 ratio. In this study, passage-3 MSCs were used for research on cell adhesion, proliferation, and differentiation. For osteogenic differentiation, MSCs were cultured in osteogenic culture medium, which was Dulbecco's modified Eagle's medium supplemented with $10 \%$ fetal bovine serum, $100 \mathrm{unit} / \mathrm{mL}$ penicillin, $100 \mu \mathrm{g} / \mathrm{mL}$ streptomycin, $50 \mathrm{~g} / \mathrm{mL}$ ascorbic acid, $10 \mathrm{mM}$ glycerophosphate, and $10^{-8} \mathrm{M}$ dexamethasone (Sigma-Aldrich).

\section{Cell viability evaluation and fluorescence microscopy observation}

The viability of MSCs cultured on the PEG/PLA hybrid fibrous scaffold was evaluated by 3-(4,5-dimethylthiazol-2-yl)-2,5diphenyltetrazolium bromide assay. The mechanism of this assay is that metabolically active cells react with a tetrazolium salt to produce a soluble formazan dye. The rat bone MSCs were cultured on the electrospun fibrous scaffolds at $37^{\circ} \mathrm{C}$ under $5 \%$ carbon dioxide for the predetermined time. Subsequently, $20 \mu \mathrm{L}$ of 3-(4,5-dimethylthiazol-2-yl)-2,5diphenyltetrazolium bromide stock solution $(5 \mathrm{mg} / \mathrm{mL})$ in phosphate buffered saline (PBS) was added to each well and the cells were further incubated for another 4 hours. The precipitated formazan was dissolved in $100 \mu \mathrm{L}$ dimethyl sulfoxide, and the absorbance at $570 \mathrm{~nm}$ was measured with spectrophotometer (Spectronic $^{\mathrm{TM}}$ 20D+; Thermo Fisher Scientific). Experiments were repeated in triplicate. 
In order to be observed by fluorescence microscopy (DM IL; Leica Microsystems, Wetzlar, Germany), the rat bone MSCs were stained by fluorescein diacetate and 2-(4-amidinophenyl)-6-indolecarbamidine dihydrochloride (Sigma-Aldrich), and cell morphology was also observed by optical microscopy.

\section{In vitro MSCs differentiation study}

Messenger ribonucleic acid (mRNA) extraction and real-time polymerase chain reaction (PCR) analysis

The total RNA of cultured MSCs was isolated using TRIzol ${ }^{\circledR}$ Reagent (Life Technologies) according to the manufacturer's protocol. Briefly, $1 \mu \mathrm{g}$ total RNA was first reversely transcribed to complementary DNA by Moloney Murine Leukemia Virus Reverse Transcriptase (Life Technologies). Then the real-time PCR reaction was carried out in a 96-well format with Taq polymerase (Thermo Fisher Scientific) and the SYBR ${ }^{\circledR}$ Premix Ex Taq ${ }^{\mathrm{TM}}$ II (Perfect Real-Time) PCR kit (Takara Biotechnology, Dalian, China) on a DNA Engine $^{\circledR}$ PTC-200 Thermal Cycler (Bio-Rad Laboratories, Hercules, CA). The primers of differentiation markers are listed in Table 1 . The levels of $\beta$-actin mRNA in each sample were used as an internal control, relative quantities were analyzed by $2^{-\Delta \Delta \mathrm{Ct}}$ method. All reactions were carried out in triplicate and the results were analyzed by Gene Expression Analysis for $\mathrm{iCycler} \mathrm{iQ}^{\circledR}$ Real-Time PCR Detection System (Bio-Rad). The products were visually analyzed on $2 \%$ agarose gel.

\section{Alkaline phosphatase (ALP) activity assay}

MSCs were cultured in osteogenic culture medium, and their ALP activity was assessed on day 4, 7, 14, and 21. The cultured cell layer was rinsed twice with PBS $1 \times$ and lysed by ALP buffer (GenMed LLC, San Diego, CA). Protein content was estimated using the Bradford assay method (GenMed). The hydrolysis of a colorless organic phosphate ester $p$-nitrophenyl phosphate to the yellow $p$-nitrophenol was catalyzed by ALP. The absorbance of the yellow product was measured at $405 \mathrm{~nm}$ using a spectrophotometer (Spectronic ${ }^{\mathrm{TM}}$ 20D+; Thermo Fisher Scientific). ALP activity was also visually stained using reagent $\mathrm{B}$ as AP-Red concentrated solution and reagent $\mathrm{A}$ as AP-Red accentuator according to the manufacturer's protocol (ZSGB-BIO, Inc, Beijing, China). The cultured cells were rinsed twice with PBS $1 \times$ and immersed in the alkaline-dye mixture for 10 minutes at room temperature. Subsequently, the dyed cells were rinsed in deionized water and investigated microscopically.

\section{Alizarin red S (ARS) staining and quantitation}

ARS staining was used to quantify the mineralization of MSCs grown on the PEG/PLA hybrid fibrous scaffold with a glass coverslip as a fiber-supporting substrate, and cells on the glass coverslip alone were included as a control. The cell-scaffold construct was washed twice with PBS $1 \times$ and fixed with $10 \%$ formaldehyde for 10 minutes. After being washed with PBS $1 \times$, the constructs were incubated with the staining solution ( $1 \%$ ARS in $2 \%$ ethanol) for 5 minutes. Subsequently, the staining solution was removed and the constructs were washed three times with distilled water to get rid of excessive color. To quantify the mineralization, the constructs were desorbed using $10 \%$ cetylpyridinium chloride (Sigma-Aldrich) for 1 hour. The solution was collected and the absorbance at $570 \mathrm{~nm}$ was measured with the spectrophotometer, which was performed in triplicate wells.

\section{Histological analysis in vivo}

To further evaluate the tissue responses to the implanted scaffold in vivo, the PEG/PLA fibrous scaffold was transplanted into the thigh muscle pouches of rats. All animal care and experimental procedures were conducted according to institutional Animal Care and Use guidelines. Briefly, the Sprague Dawley rats (4 weeks old, $200 \pm 20 \mathrm{~g}$ ) were first anesthetized with intraperitoneal injection of chloral hydrate $(0.3 \mathrm{~mL} / 100 \mathrm{~g})$ until effectively anesthetized. Subsequently, the PEG/PLA fibrous scaffold was transplanted into the thigh muscle pouches. Three rats were randomly sacrificed

Table I Nucleotide primers used for reverse transcription polymerase chain reaction

\begin{tabular}{|c|c|c|c|c|}
\hline Gene & Primer sequences & Annealing & Product size (bp) & GenBank accession number \\
\hline \multirow[t]{2}{*}{ Cbfa-I } & F 5'-ATGCGTATTCCCGTAGATC & $56^{\circ} \mathrm{C}$ & 487 & NM_053470.I \\
\hline & R 5'-GTAGCTCGGCAGAGTAGTTC & & & \\
\hline \multirow[t]{2}{*}{ Coll } & F 5'-TGGATGGCTGCACGAGT & $56^{\circ} \mathrm{C}$ & 177 & NM_053304.I \\
\hline & R 5'-TTGGGATGGAGGGAGTTTA & & & \\
\hline \multirow[t]{2}{*}{ OCN } & F 5'-AGGGCAGTAAGGTGGTGAA & $56^{\circ} \mathrm{C}$ & 143 & NM_0I34I4.I \\
\hline & R 5'-GCCAATGTGGTCCGCTA & & & \\
\hline \multirow[t]{2}{*}{ OPN } & F 5'-CAGCAGGACTGAAGGAGC & $55^{\circ} \mathrm{C}$ & 148 & NM_0I288I.2 \\
\hline & R 5'-GGAGACAGGAGGCAAGG & & & \\
\hline
\end{tabular}


at predetermined time of 2 weeks and 4 weeks, and the excised implant and surrounding muscle were fixed in $10 \%$ formalin for histological analysis. The fixed specimens were serially dehydrated in a graded series of ethanol washes and embedded in paraffin, then eight micron-thick sections were cut and stained according to standard protocols for hematoxylin and eosin staining. All hematoxylin and eosin results were analyzed with a digital image analysis system (Eclipse E600 microscope with DXM 1200 digital camera; Nikon Corporation, Tokyo, Japan).

\section{Statistics}

Statistical analysis was performed using the GraphPad Prism (v 5.0; GraphPad Software, Inc, La Jolla, CA) and the statistical significance of the difference between groups was found by one-way analysis of variance. Quantitative data were represented as mean \pm standard deviation and statistical significance was set at $P<0.05$.

\section{Results \\ Morphology analysis of the electrospun PEG/PLA fibrous scaffolds by SEM}

The morphology of the electrospun fibrous scaffolds was observed by SEM (Figure 1). Under optimized electrospinning conditions (eg, solvent, solution concentration), PEG/ PLA (PEG/PLA = 5/95, optimized by the authors, data not shown) hybrid solution was electrospun to form smooth uniform fibers (Figure 1C and D). The average diameter of the PEG/PLA electrospun fibers obtained was $2.01 \pm 0.32 \mu \mathrm{m}$, which was one to two orders of magnitude smaller than mammalian cells, and included the range of feature sizes
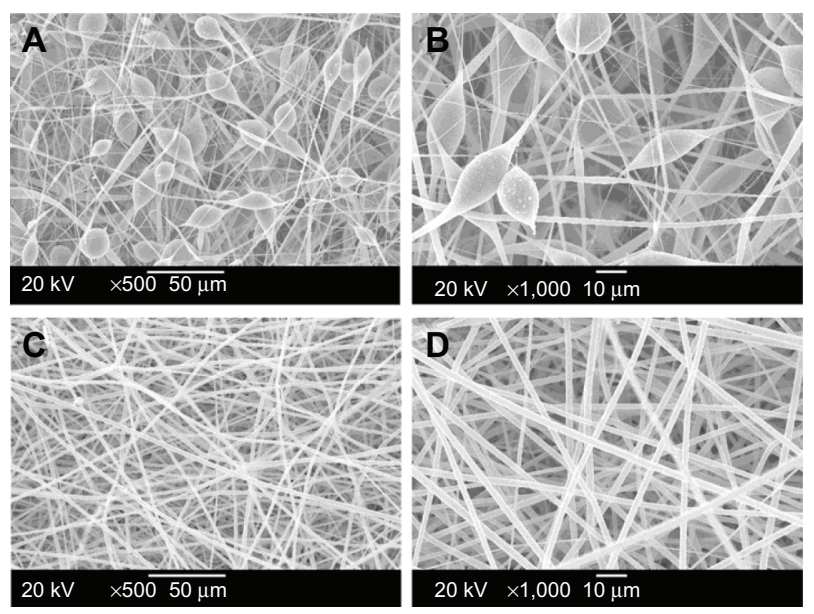

Figure I Scanning electron microscope images of the electrospun pure polylactide (PLA) and poly(ethylene glycol) (PEG)/PLA hybrid membrane. (A) Pure PLA, 500x; (B) pure PLA, 1000x; (C) PEG/PLA, 500x; and (D) PEG/PLA, 1000×. known to facilitate contact guidance. ${ }^{39}$ For pristine PLA fiber mats as presented in Figure $1 \mathrm{~A}$ and $\mathrm{B}$, the diameter of the electrospun fibers had a broad distribution ranging from $200 \mathrm{~nm}$ to $5 \mu \mathrm{m}$, some with spindle-shaped structures. The irregular and discontinuous fibers may be due to the intrinsic properties of PLA solution such as hydrophobicity, viscosity, and surface tension. ${ }^{28,29}$ The spun PLA/PEG fibers were long and continuous, which may be attributed to the reduction of solution viscosity caused by the use of the low molecular weight PEG as lubricant.

\section{Cell behavior of MSCs on the PEG/PLA fibrous scaffolds}

Cell morphology and spreading, as well as cell interaction with the electrospun fibrous scaffolds were studied by SEM (Figure 2). On the day following cell seeding (Figure 2A and B), MSCs had already expanded on the surface of the electrospun fibrous scaffolds, and the anchoring ligands of the cells had stretched out to elongate along individual fibers thinner than themselves. Meanwhile, the seeded cells were thin and narrow in shape, and adhered to a relatively small area of the scaffold. ${ }^{40}$ Subsequently, MSCs started to migrate through the pores and integrated well with the surrounding fibers on day 3 (Figure 2C and D). Moreover, it
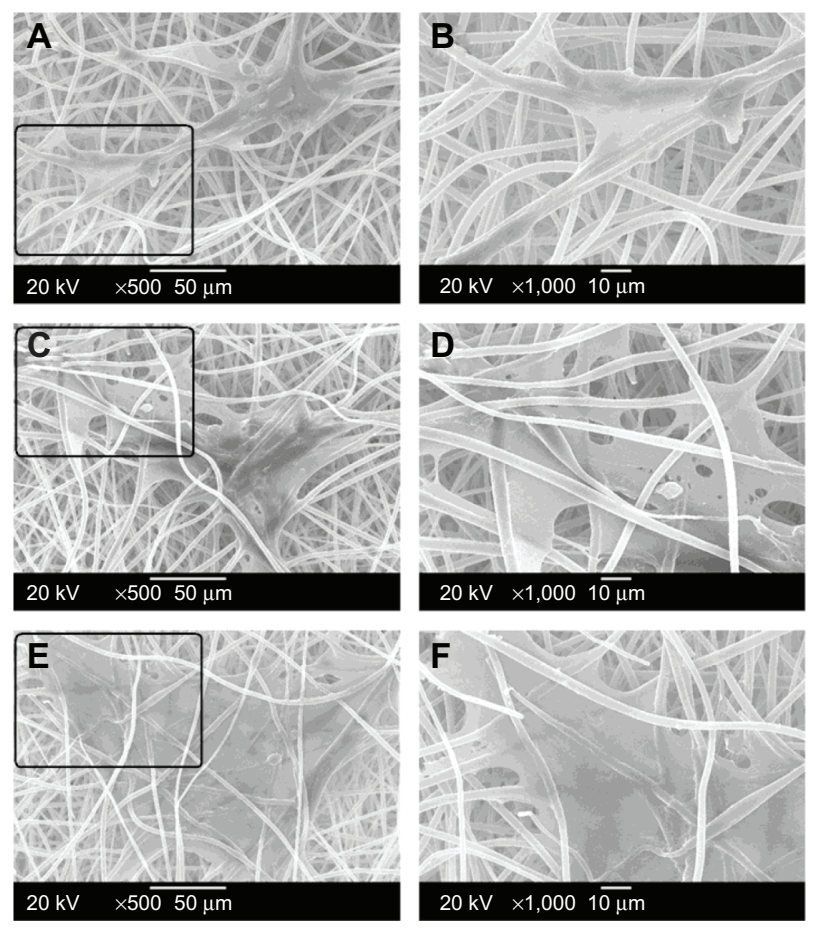

Figure 2 Scanning electron microscope observations of rat bone mesenchymal stem cell adhesion and growth on the electrospun poly(ethylene glycol)/polylactide hybrid membranes for I day (A, B), 3 days (C, D), and 5 days (E, F) at different magnification (A, C, E: 500x; B, D, F: 1000x). 
became extremely difficult to determine the exact boundary of a single cell since the cells grew massively and formed a continuous layer. MSCs on the fibrous scaffolds expanded even more and almost reached confluence after 5 days in culture (Figure 2E and F). Interestingly, MSCs began to penetrate into the fibrous scaffold through the interstitial pores between the fibers and grow underneath the fiber network from day 3 . The penetration of cells into the fibrous scaffolds was significantly meaningful for the cytocompatibility evaluation and very important for favorable application in tissue engineering. This is because the functional tissue can only be regenerated when cells migrate through the scaffolds, but not when they just stay on the scaffold surface. ${ }^{41}$

Cell viability and metabolic activity of MSCs cultured on the PEG/PLA electrospun fibrous scaffolds showed a statistically significant increase compared with those on the tissue culture plate 1 day and 3 days after seeding (Figure 3 ). However, the cells on the scaffolds proliferated slightly up to day 5 when the MSCs began to differentiate, which is in accordance with osteoblast proliferation and differentiation having conflicting properties. ${ }^{42}$ The higher cell viability for the PEG/PLA electrospun fibrous scaffolds demonstrates that the electrospinning scaffolds can accelerate initial attachment of the cells and promote cells to penetration into fiber substrates, which is important for the application of biomaterial scaffolds. ${ }^{4,33}$ In order to further confirm cytocompatibility of the PEG/PLA electrospun fibrous scaffolds, fluorescent microscopic images of MSCs grown on the scaffolds were taken. As shown in Figure 4, the cells were observed to adhere

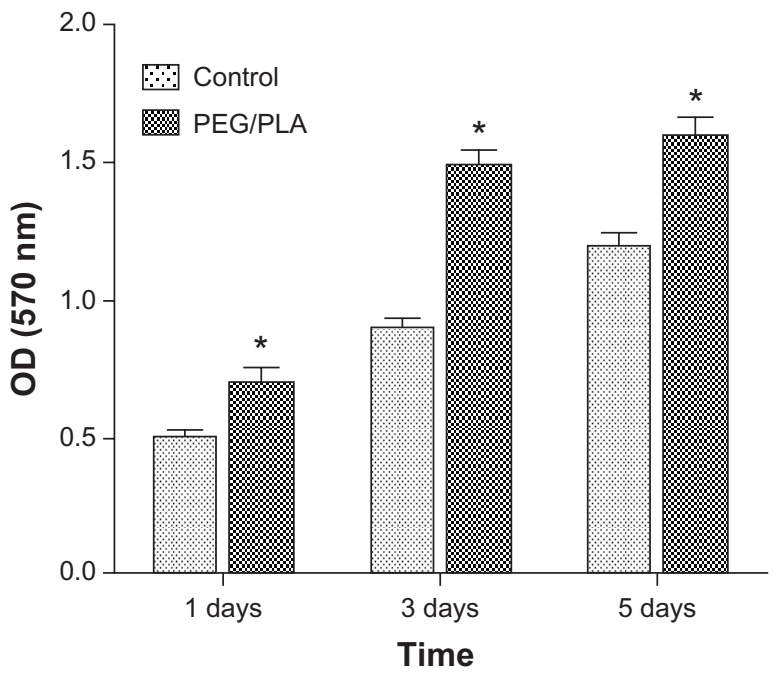

Figure 3 Mesenchymal stem cells viability and metabolic activity evaluation on the poly(ethylene glycol)/polylactide hybrid membranes, with the tissue culture plate as the control.

Note: Error bars represent standard deviation; $\mathrm{n}=3$.

Abbreviations: PEG/PLA, poly(ethylene glycol)/polylactide; OD, optical density.
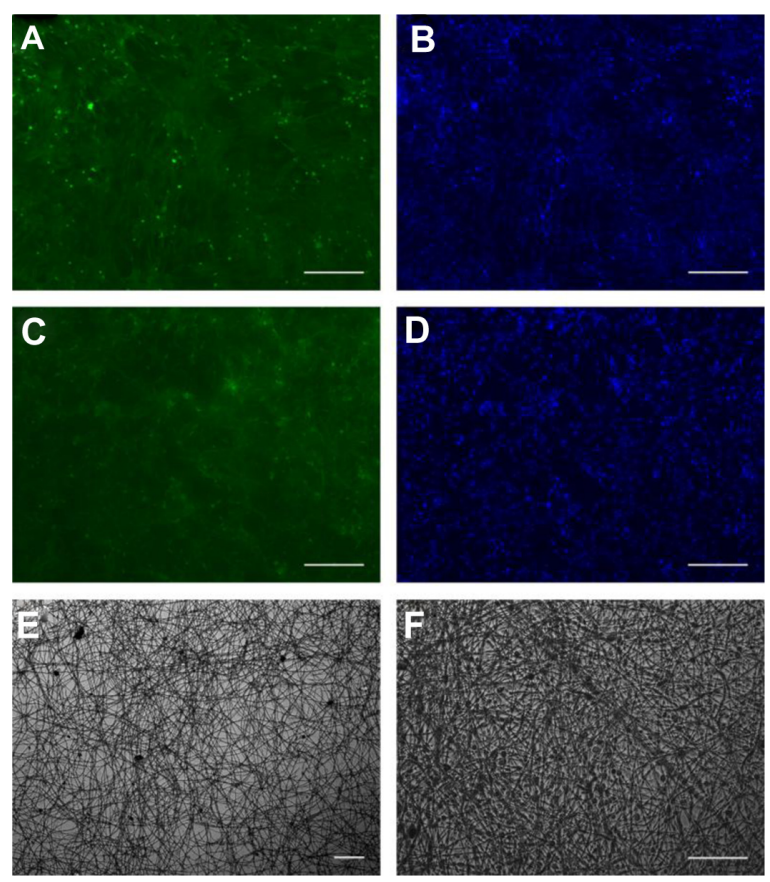

Figure 4 Fluorescence micrographs show the cytocompatibility of the poly(ethylene glycol)/polylactide electrospun fibrous scaffolds with mesenchymal stem cells (C, D). Mesenchymal stem cells cultured on the tissue culture plate were the control $(\mathbf{A}, \mathbf{B})$. The cells were stained with fluorescein diacetate in green color $(\mathbf{A}, \mathbf{C})$ and 2-(4-amidinophenyl)-6-indolecarbamidine dihydrochloride for cell nuclei in blue (B, D). Mesenchymal stem cells on the poly(ethylene glycol)/polylactide electrospun fibrous scaffolds under white light were also included at different magnification (E, F).

Note: Scale bar $=100 \mu \mathrm{m}$.

and spread actively with a physiological adherence pattern on both the tissue culture plate and the fibrous scaffolds.

\section{MSCs osteogenic development on the PEG/PLA fibrous scaffolds}

In addition to attachment and proliferation, the ability to support cultured cell differentiation is another actual applicability of the scaffold. By analyzing the genes of different clusters and their expression profiles, there were three main phases of osteogenic development procedure as reported: proliferation, matrix maturation, and mineralization, ${ }^{43}$ as shown in Figure 5.

Gene expression profile during osteogenic procedure To confirm the osteogenic differentiation of MSCs on the PEG/PLA fibrous scaffolds, induction of osteoblast-specific

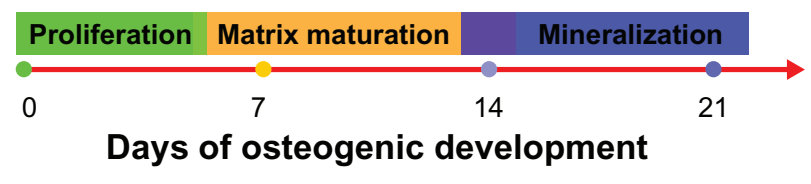

Figure 5 Graphical summary of the three phases and time schedule of osteogenic induction: proliferation, matrix maturation, and mineralization. 
genes was examined by real-time polymerase chain reaction (PCR) (Figure 6). As the earliest osteogenic marker, the transcriptional factor $C b f a-1$ drove the pluripotent MSCs to preosteoblasts by initiating the expression of many bone specific genes. ${ }^{44} \mathrm{Col} I$ comprised $90 \%-95 \%$ of the organic material of mineralized ECM and served as a template upon which mineral deposited, which was well known as one of the mid-stage osteogenic markers. ${ }^{45}$ The expression of Cbfa- 1 and $\mathrm{Col} I$ was upregulated during the whole period of differentiation; maximum expression was reached on day 21 . Furthermore, the expression of $\mathrm{Cbfa}-\mathrm{I}$ and $\mathrm{Col} \mathrm{I}$ of MSCs on the scaffolds was significantly higher than those on the glass coverslip throughout differentiation. The maturation of differentiation was accomplished with the secretion of the late osteogenic differentiation markers OCN (osteocalcin) and OPN (osteopontin). After 14 days of osteogenic induction, the secreted $O C N$ and $O P N$ on the scaffolds showed much higher expression compared with the control. By evaluating the expression of the osteoblast phenotypic markers, MSCs cultured on the PEG/PLA fibrous scaffolds presented better osteogenic differentiation ability.

\section{ALP activity analysis}

As a well-known marker of the osteogenic phenotype, ALP is a glycoprotein on the cell membrane that catalyzes the hydrolysis of phosphate esters at an alkaline $\mathrm{pH}$ and plays an important

A

\begin{tabular}{|c|c|c|c|c|c|c|}
\hline & \multicolumn{2}{|c|}{7 days } & \multicolumn{2}{|c|}{14 days } & \multicolumn{2}{|c|}{21 days } \\
\hline & a & $b$ & a & $b$ & a & b \\
\hline Cbfa1 & 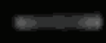 & $\infty$ & $=$ & $=$ & $=$ & $\infty$ \\
\hline Col 1 & $\mathrm{Bec}$ & 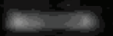 & 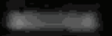 & $\mathrm{Ba}$ & 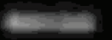 & $=$ \\
\hline OCN & 8 & 4 & 25 & $2=$ & $=$ & $\mathrm{n}$ \\
\hline OPN & bis & $\alpha=50$ & $\infty=$ & 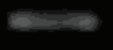 & $=$ & - \\
\hline$\beta$-actin & - & - & 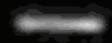 & -1 & 0 & $=$ \\
\hline
\end{tabular}

B
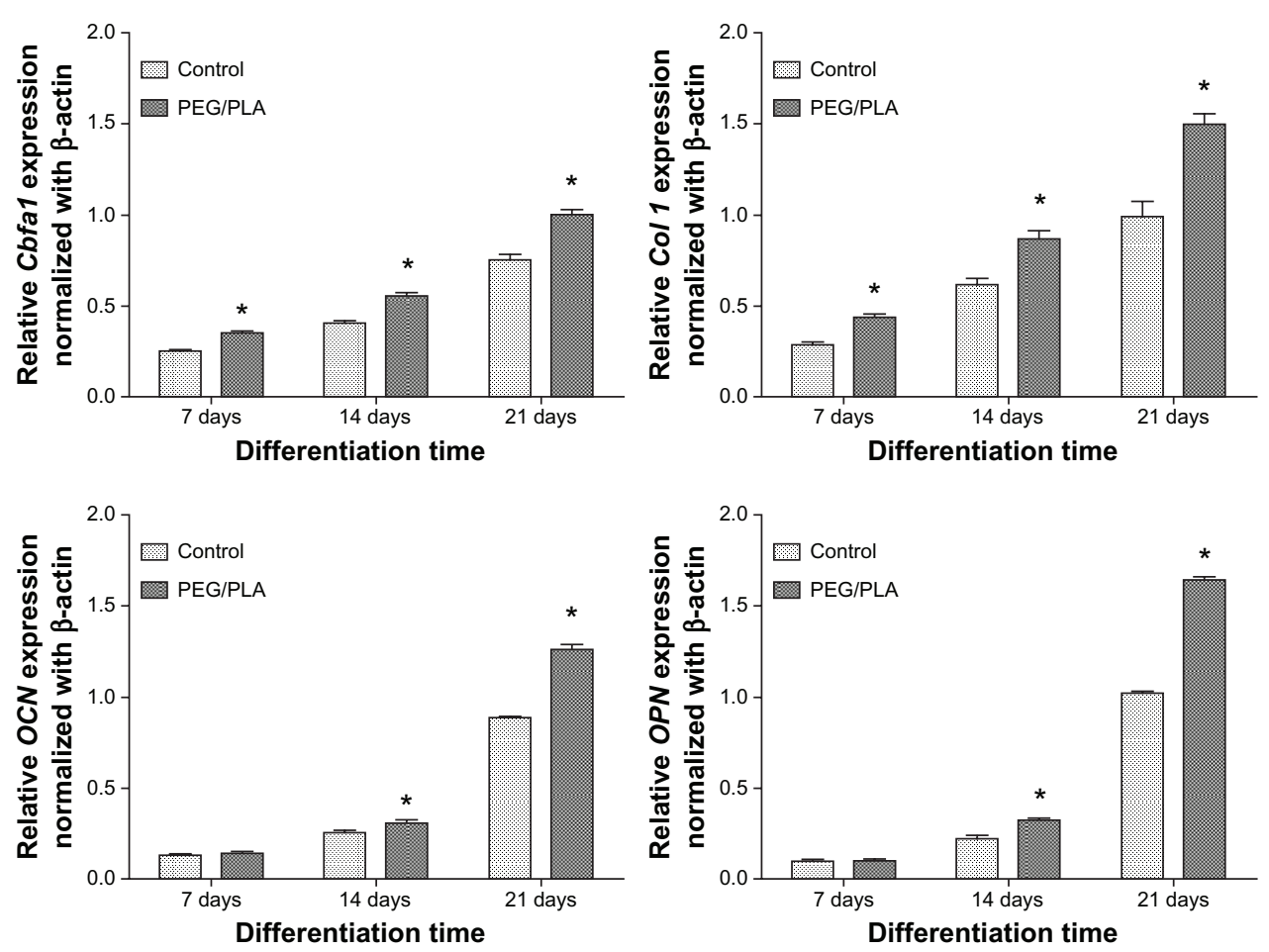

Figure 6 Gene expression profile of rat bone mesenchymal stem cells in osteogenic cultures. (A) Agarose gel electrophoresis result of the real-time polymerase chain reaction product shows the effects of the culture scaffold on gene expression on day 7, I4, and 2I. (B) Quantitative analysis for Col I, Cbfa-I, OCN, and OPN expression was performed by Gene Expression Analysis for iCycler iQ ${ }^{\circledR}$ Real-Time PCR Detection System (Bio-Rad Laboratories, Hercules, CA); relative gene expression levels were normalized by the housekeeping gene, $\beta$-actin.

Note: *Represents statistically significant difference $(P<0.05), \mathrm{n}=3$.

Abbreviation: PEG/PLA, poly(ethylene glycol)/polylactide. 
role in bone matrix mineralization process ${ }^{46}$ In this study, ALP activity was visualized by ALP staining (Figure 7B and C) on day 14 and evaluated quantitatively in terms of $\mu \mathrm{mol}$ of $p$-nitrophenyl phosphate production per mg of protein per hour (Figure 7A) on day 4, 7, 14, and 21. ALP expression is known to peak after the end of the proliferative stage and before matrix maturation during osteoblastic differentiation ${ }^{47}$ As presented in Figure 7A, ALP activity of MSCs cultured both on glass coverslip and fibrous scaffolds presented an ongoing increase with culture time up to 14 days and then a decrease on day 21, which suggested that osteodifferentiation was occurring. Additionally, ALP activity was significantly higher for the PEG/PLA fibrous scaffolds than the control from day 7 , which indicated that the presence of the bone-like matrix enhanced differentiation from MSCs to osteoblast-like cells.

\section{ARS staining and mineralization quantification}

The ARS dye binds to calcium salts selectively and is widely used for calcium mineral histochemistry. Figure $8 \mathrm{~A}$ and B present the images of ARS staining of MSCs cultured on glass coverslip and PEG/PLA fibrous scaffolds on day 21 in osteogenic culture medium. The red stains (bone nodules) were ARS-calcium chelating products. ${ }^{48}$ As shown in Figure 8B, the cells cultured on PEG/PLA fibrous scaffolds clearly showed matrix mineralization with more intense ARS
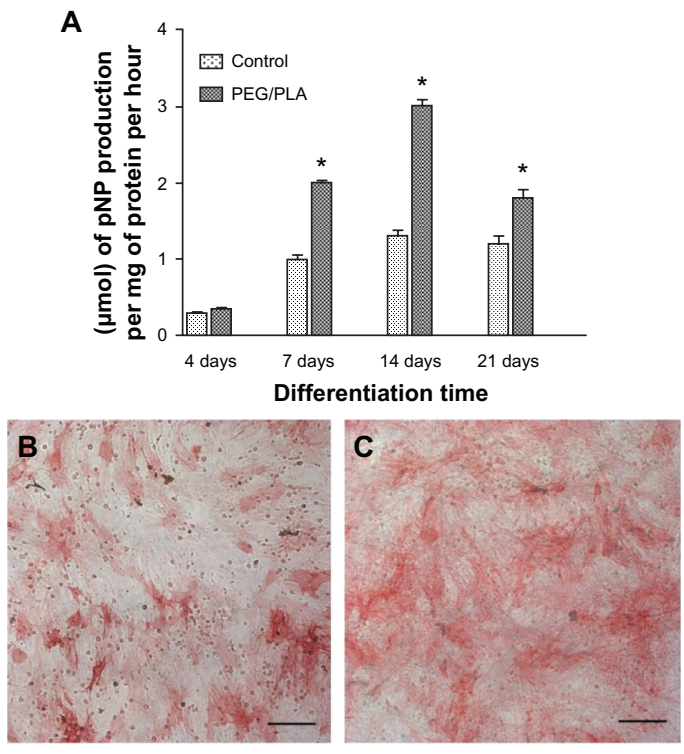

Figure 7 Alkaline phosphatase activity expressed by the cells after culturing for 4, 7, 14 , and $2 \mathrm{I}$ days. A glass coverslip was used as a fiber-supporting substrate and data on the glass coverslip was included as a control. (A) Alkaline phosphatase activity quantitative evaluation on day 4, 7, I4, and 21 . Alkaline phosphatase staining was also performed on control (B) and poly(ethylene glycol)/polylactide hybrid scaffolds (C) on day 14 .

Notes: Scale bar $=50 \mu \mathrm{m} .{ }^{*}$ Represents statistically significant difference $(P<0.05)$, $\mathrm{n}=3$.

Abbreviations: PEG/PLA, poly(ethylene glycol)/polylactide; pNP, p-nitrophenyl phosphate.
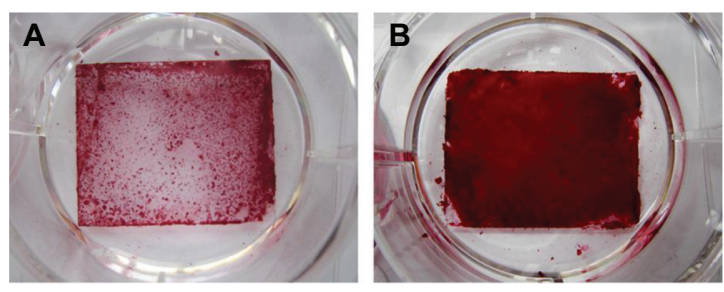

C

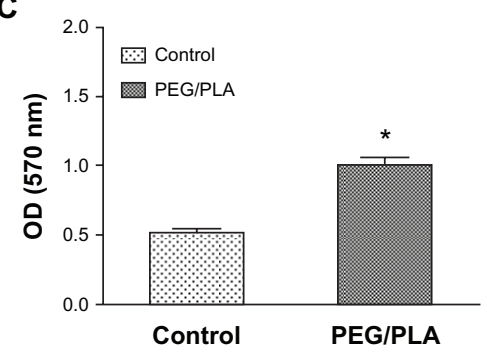

Figure 8 Analysis of the mineral deposition in osteogenic mesenchymal stem cells on day 21 by Alizarin Red S staining. Photographs of the Alizarin Red S staining show higher calcium deposition of the poly(ethylene glycol)/polylactide fibrous scaffolds with a glass coverslip as the fiber-supporting substrate (A) than on the glass coverslip as control (B). The quantification result confirmed the higher Alizarin Red S staining of the poly(ethylene glycol)/polylactide fibrous scaffolds (C).

Note: *Represents statistically significant difference $(P<0.05), \mathrm{n}=3$.

Abbreviations: PEG/PLA, poly(ethylene glycol)/polylactide; OD, optical density.

staining than the control. To quantify mineralization, constructs were desorbed using $10 \%$ cetylpyridinium chloride for 1 hour, and the absorbance of the solution was measured at $570 \mathrm{~nm}(\mathrm{n}=3)$. The results of the absorbance analysis quantitatively confirmed the higher calcium deposition of the PEG/PLA fibrous scaffolds (Figure 8C, $P<0.05$ ).

On the whole, the PEG/PLA fibrous scaffolds presented good cellular compatibility and excellent osteogenic potential in vitro. Therefore, they could be one of the most promising candidates for potential application in tissue engineering.

\section{Histologic analysis of tissue response to the PEG/PLA fibrous scaffolds}

The PEG/PLA fibrous scaffold was transplanted into the thigh muscle pouches of rats, and the histologic results were analyzed to evaluate the tissue responses in vivo. The host response was determined by measuring the inflammatory cells, ie, macrophage, polymorphonuclear leukocyte, lymphocyte, plasma cell, and giant cell. As depicted in Figure 9A, an acute inflammation reaction occurred at week 2, and the inflammatory cells infiltrated into the PEG/PLA fibrous scaffold $\left(3815 \pm 317 / \mathrm{mm}^{2}\right)$. However, the inflammation reaction attenuated dramatically and the number reduced to $201 \pm 84 / \mathrm{mm}^{2}$ at 4 weeks (Figure 9B). ${ }^{5}$ Meanwhile, the surrounding muscle maintained its physiological characteristics the whole time, as confirmed by both macroscopic observation (data not shown) and histologic analysis 

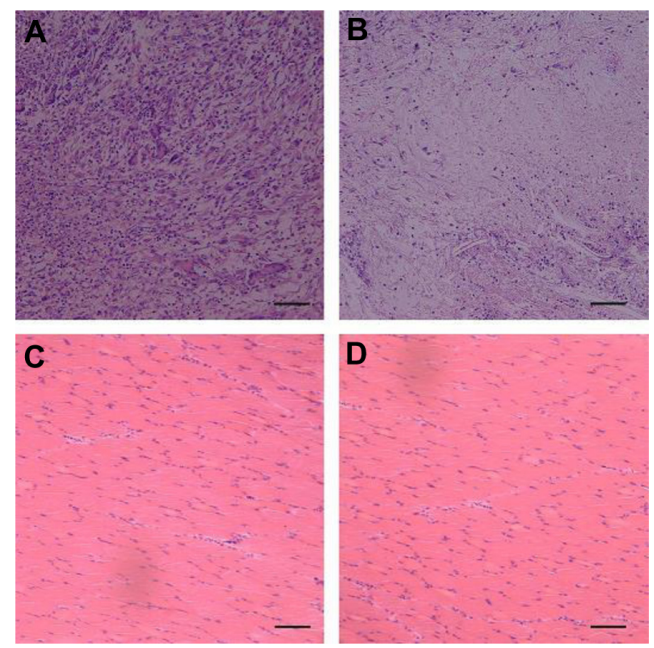

Figure 9 Histologic analysis of tissue response to poly(ethylene glycol)/polylactide fibrous scaffolds. There was a dense accumulation of inflammatory cells presented around the poly(ethylene glycol)/polylactide fibrous scaffolds at 2 weeks (A) but attenuated at week 4 (B). Meanwhile, the surrounding muscle presented good physiological characteristics both at 2 weeks (C) and 4 weeks (D).

Note: Scale bar $=50 \mu \mathrm{m}$

(Figure 9C and D). In general, the PEG/PLA fibrous scaffold presented good biocompatibility in vivo.

\section{Discussion}

When used as bone tissue engineering scaffolds, electrospun fibers offer a range of attractive features such as large surface area-to-volume ratios, interconnected porous structures, ease of construction into different shapes, and similar structures to natural ECM. The advantages mentioned above made the electrospun fibers ideal for cell adhesion and proliferation as well as for nutrient and metabolic waste exchange. Meanwhile, bone marrow MSCs are multipotent cells that have been considered as ideal seed cells in the bone repair. ${ }^{49}$ Therefore, the aim in the present study was to evaluate the cell response of obtained PEG/PLA electrospun hybrid scaffolds to MSCs, and the osteogenic development behavior of MSCs cultured on the PEG/PLA electrospun fibrous scaffold in vitro. Surrounding tissue response to the fibrous scaffolds in vivo was also evaluated by transplanting the PEG/PLA electrospun hybrid scaffold into the thigh muscle pouches of rats.

As shown by SEM, the average diameter of the uniform and continuous hybrid PEG/PLA fibrous scaffolds was one to two orders of magnitude smaller than mammalian cells, which included the range of feature sizes to facilitate contact guidance. ${ }^{33}$ The diameter of the pure PLA electrospun fibers had a broad distribution ranging from $200 \mathrm{~nm}$ to $5 \mu \mathrm{m}$, some with spindle-shaped structures. It was presumed that hybrid PEG/PLA fibrous scaffolds would present good cell response and facilitate osteoblastic differentiation.
To validate the cell response of PEG/PLA fibrous scaffolds, the behavior of MSCs cultured on the scaffolds, including the cell morphology and cell interaction with the electrospun fibrous scaffolds by SEM, cell viability, and metabolic activity by 3-(4,5-dimethylthiazol-2-yl)2,5-diphenyltetrazolium bromide accompanied with the fluorescent microscopic images, were evaluated. MSC behavior confirmed the presumption that MSCs interacted and integrated well with surrounding fibers. Moreover, MSCs attached and proliferated better on the scaffolds than on the glass coverslip. As reported, the cells gradually penetrated into the fibrous structures composed of large diameter fibers due to the large pore area of the fibrous structures, but failed to penetrate into the fibrous structures composed of small diameter fibers. ${ }^{27}$ As the interstitial pore sizes of obtained PEG/PLA fibrous scaffolds were larger than $10 \mu \mathrm{m}$, it was possible for MSCs to fully penetrate into the fibrous scaffolds through these pores. MSCs on the scaffolds formed a continuous layer and even migrated through the pores to grow underneath the fiber network, which is significant for favorable application in tissue engineering as the functional tissue would be regenerated through the scaffolds and not just on the scaffold surface.

In addition to attachment and proliferation, the osteoinductive capacity of a scaffold to support differentiation of cultured cells is another important aspect suggesting actual applicability of the scaffold. Of the several kinds of osteogenic cells, bone marrow MSCs seem to be the optimal seeding cells for bone tissue engineering owing to the advantages mentioned above. As briefly depicted in Figure 5, there were three main phases of osteogenic development: proliferation, matrix maturation, and mineralization. As reported, the blending of PEG to PLA can modulate the hydrophilicity property and significantly enhance osteoblastic differentiation. ${ }^{33,34}$ Representative markers of the osteogenic procedure were comprehensively analyzed by mRNA quantitation and protein analysis (Figures 6-8). As the earliest osteogenic marker, mRNA level of $C b f a-1$ was upregulated during the whole differentiation period and reached maximum expression on day 21 . The transcriptional factor $C b f a-1$ can drive the pluripotent MSCs to preosteoblasts by initiating the expression of many bone specific genes, so the higher expression of $C b f a-1$ for MSCs on the PEG/PLA fibrous scaffolds presented better differentiation potential. The well-known mid-stage osteogenic markers were Col I and ALP. The higher Col I mRNA level provided a favorable template for the mineral deposition for the reason 
that the Col I comprised $90 \%-95 \%$ of the organic material of mineralized ECM. The bone-forming potential of MSCs was further analyzed by evaluating the expression of ALP activity, since ALP is regarded to be an important phenotype of bone-forming cells. Of particular note, ALP activity of MSCs at all evaluation times was significantly higher on the PEG/PLA fibrous scaffolds than on the pure glass coverslip (Figure 7). At the mineralization stage of differentiation, the maturation of the osteogenic induction was confirmed by the high expression of the late osteogenic differentiation markers $O C N$ and $O P N$ accompanied with positive ARS staining (Figure 8). In accordance with previous results, the expression of the late osteogenic differentiation markers confirmed the presumption that the PEG/PLA fibrous scaffolds is better at encouraging cells to differentiate into bone-associated cells in vitro, which will ultimately be useful as bone constructs in bone tissue engineering.

Based on the good cellular compatibility and excellent osteogenic potential, an experiment on surrounding tissue response to the PEG/PLA fibrous scaffolds in vivo was briefly performed. Acute inflammation reaction presented at 2 weeks, but attenuated at week 4 without unfavorable influence on the ambient muscle, which indicated that the PEG/ PLA fibrous scaffolds can get along well with surrounding tissues in vivo.

On the whole, the obtained PEG/PLA fibrous scaffolds could be one of the best potential bone implant candidates in bone tissue engineering. However, to confirm the practical usefulness of this novel PEG/PLA hybrid fibrous scaffold, more in-depth studies on the hybridized fibers are under way, in particular animal tests to find clinical applications such as guided bone regeneration and tissue engineering matrices.

\section{Conclusion}

In this study, uniform PEG/PLA fibrous scaffolds were successfully prepared by electrospinning and they exhibited improved morphology with regular and continuous fibers compared to corresponding pristine PLA fiber mats. The obtained PEG/PLA fibrous scaffolds favored MSC attachment and proliferation, furthermore, MSCs penetrated into the fibrous scaffold through the interstitial pores which was meaningful for cytocompatibility evaluation. After comprehensively analyzing the representative markers of the osteogenic procedure at mRNA level and protein level in vitro, as well as the histologic result of the tissue responses in vivo, it can be concluded that the prepared PEG/PLA fibrous scaffolds present excellent osteogenic potential and prospective use for bone tissue engineering.

\section{Acknowledgments}

This work was financially supported by the National 863 project (2007 AA021902) and New Century Excellent Talents in University (NCET-08-0371). We would like to give warm thanks to Wang Hui (Analytical and Testing Center, Sichuan University) for her great help in SEM analysis and Ge Yan (Regenerative Medicine Research Center, West China Hospital, Sichuan University) for her kind guidance in the preparation of MSCs.

\section{Disclosure}

The authors report no conflict of interest in this work. The authors are solely responsible for the content and writing of the paper.

\section{References}

1. Dong SW, Ying DJ, Duan XJ, et al. Bone regeneration using an acellular extracellular matrix and bone marrow mesenchymal stem cells expressing Cbfa1. Biosci Biotechnol Biochem. 2009;73(10):2226-2233.

2. Lutolf MP, Hubbell JA. Synthetic biomaterials as instructive extracellular microenvironments for morphogenesis in tissue engineering. Nat Biotechnol. 2005;23(1):47-55.

3. Martins A, Chung S, Pedro AJ, et al. Hierarchical starch-based fibrous scaffold for bone tissue engineering applications. J Tissue Eng Regen Med. 2009;3(1):37-42.

4. Fang R, Zhang E, Xu L, Wei S. Electrospun PCL/PLA/HA based nanofibers as scaffold for osteoblast-like cells. J Nanosci Nanotechnol. 2010;10(11):7747-7751.

5. Munch E, Launey ME, Alsem DH, Saiz E, Tomsia AP, Ritchie RO. Tough, bio-inspired hybrid materials. Science. 2008;322(5907):1516-1520.

6. Pham QP, Sharma U, Mikos AG. Electrospinning of polymeric nanofibers for tissue engineering applications: a review. Tissue Eng. 2006; 12(5):1197-1211.

7. Kang YM, Kim KH, Seol YJ, Rhee SH. Evaluations of osteogenic and osteoconductive properties of a non-woven silica gel fabric made by the electrospinning method. Acta Biomater. 2009;5(1):462-469.

8. Rouwkema J, Rivron NC, van Blitterswijk CA. Vascularization in tissue engineering. Trends Biotechnol. 2008;26(8):434-441.

9. Zeleny J. The electrical discharge from liquid points, and a hydrostatic method of measuring the electric intensity at their surfaces. Phys Rev. 1914;3(2):69-91.

10. Formhals A, inventor. Process and apparatus for preparing artificial threads. United States patent 1975 504. 1934.

11. Xie J, Li X, Xia Y. Putting electrospun nanofibers to work for biomedical research. Macromol Rapid Commun. 2008;29(22):1775-1792.

12. He W, Yong T, Teo WE, Ma Z, Ramakrishna S. Fabrication and endothelialization of collagen-blended biodegradable polymer nanofibers: potential vascular graft for blood vessel tissue engineering. Tissue Eng. 2005;11(9-10):1574-1588.

13. Heydarkhan-Hagvall S, Schenke-Layland K, Dhanasopon AP, et al. Three-dimensional electrospun ECM-based hybrid scaffolds for cardiovascular tissue engineering. Biomaterials. 2008;29(19):2907-2914.

14. Dzenis Y. Material science. Spinning continuous fibers for nanotechnology. Science. 2004;304(5679):1917-1919.

15. Chiu JB, Liu C, Hsiao BS, Chu B, Hadjiargyrou M. Functionalization of poly(L-lactide) nanofibrous scaffolds with bioactive collagen molecules. J Biomed Mater Res A. 2007;83(4):1117-1127.

16. Wutticharoenmongkol P, Pavasant P, Supaphol P. Osteoblastic phenotype expression of MC3T3-E1 cultured on electrospun polycaprolactone fiber mats filled with hydroxyapatite nanoparticles. Biomacromolecules. 2007;8(8):2602-2610. 
17. Meechaisue C, Wutticharoenmongkol P, Waraput R, et al. Preparation of electrospun silk fibroin fiber mats as bone scaffolds: a preliminary study. Biomed Mater. 2007;2(3):181-188.

18. Xin X, Hussain M, Mao JJ. Continuing differentiation of human mesenchymal stem cells and induced chondrogenic and osteogenic lineages in electrospun PLGA nanofiber scaffold. Biomaterials. 2007; 28(2):316-325.

19. Shih YR, Chen CN, Tsai SW, Wang YJ, Lee OK. Growth of mesenchymal stem cells on electrospun type I collagen nanofibers. Stem Cells. 2006;24(11):2391-2397.

20. Kim KH, Jeong L, Park HN, et al. Biological efficacy of silk fibroin nanofiber membranes for guided bone regeneration. J Biotechnol. 2005; 120(3):327-339.

21. Lee $\mathrm{CH}$, Shin $\mathrm{HJ}$, Cho $\mathrm{IH}$, et al. Nanofiber alignment and direction of mechanical strain affect the ECM production of human ACL fibroblast. Biomaterials. 2005;26(11):1261-1270.

22. Riboldi SA, Sampaolesi M, Neuenschwander P, Cossu G, Mantero S Electrospun degradable polyesterurethane membranes: potential scaffolds for skeletal muscle tissue engineering. Biomaterials. 2005;26(22): 4606-4615.

23. Nie H, Soh BW, Fu YC, Wang CH. Three-dimensional fibrous PLGA/ HAp composite scaffold for BMP-2 delivery. Biotechnol Bioeng. 2008; 99(1):223-234.

24. Jiang H, Fang D, Hsiao BS, Chu B, Chen W. Optimization and characterization of dextran membranes prepared by electrospinning. Biomacromolecules. 2004;5(2):326-333.

25. Ouchi T, Ichimura S, Ohya Y. Synthesis of branched poly(lactide) using polyglycidol and thermal, mechanical properties of its solution-cast film. Polymer. 2006;47(1):429-434.

26. Cui YL, Qi AD, Liu WG, et al. Biomimetic surface modification of poly(L-lactic acid) with chitosan and its effects on articular chondrocytes in vitro. Biomaterials. 2003;24(21):3859-3868.

27. JuYM, Choi JS, Atala A, Yoo JJ, Lee SJ. Bilayered scaffold for engineering cellularized blood vessels. Biomaterials. 2010;31(15):4313-4321.

28. Zong XH, Kim K, Fang DF, Ran SF, Hsiao BS, Chu B. Structure and process relationship of electrospun bioabsorbable nanofiber membranes. Polymer. 2002;43(16):4403-4412.

29. Deitzel JM, Kleinmeyer J, Harris D, Beck Tan NC. The effect of processing variables on the morphology of electrospun nanofibers and textiles. Polymer. 2001;42(1):261-272.

30. Stephansson SN, Byers BA, Garcia AJ. Enhanced expression of the osteoblastic phenotype on substrates that modulate fibronectin conformation and integrin receptor binding. Biomaterials. 2002;23(12): 2527-2534.

31. Lin Y, Wang L, Zhang P, et al. Surface modification of poly(L-lactic acid) to improve its cytocompatibility via assembly of polyelectrolytes and gelatin. Acta Biomater. 2006;2(2):155-164.

32. Sheth M, Ananda Kumar R, Dave V, Gross RA, McCarthy SP. Biodegradable polymer blends of poly(lactic acid) and poly(ethylene glycol). J Appl Polym Sci. 1997;66(8):1495-1505.

33. Badami AS, Kreke MR, Thompson MS, Riffle JS, Goldstein AS. Effect of fiber diameter on spreading, proliferation, and differentiation of osteoblastic cells on electrospun poly(lactic acid) substrates. Biomaterials. 2006;27(4):596-606.
34. Lieb E, Tessmar J, Hacker M, et al. Poly(D,L-lactic acid)-poly(ethylene glycol)-monomethyl ether diblock copolymers control adhesion and osteoblastic differentiation of marrow stromal cells. Tissue Eng. 2003;9(1):71-84.

35. Kim SJ, Kim MR, Oh JS, Han I, Shin SW. Effects of polycaprolactone-tricalcium phosphate, recombinant human bone morphogenetic protein-2 and dog mesenchymal stem cells on bone formation: pilot study in dogs. Yonsei Med J. 2009;50(6):825-831.

36. Murdoch AD, Grady LM, Ablett MP, Katopodi T, Meadows RS, Hardingham TE. Chondrogenic differentiation of human bone marrow stem cells in transwell cultures: generation of scaffold-free cartilage. Stem Cells. 2007;25(11):2786-2796.

37. Institute of Laboratory Animal Resources. Guide for the Care and Use of Laboratory Animals. Washington, DC: National Academies Press; 1996.

38. Kim HS, Lee HJ, Yeu IS, Yi JS, Yang JH, Lee IW. The neovascularization effect of bone marrow stromal cells in temporal muscle after encephalomyosynangiosis in chronic cerebral ischemic rats. $J$ Korean Neurosurg Soc. 2008;44(4):249-255.

39. Singhvi R, Stephanopoulos G, Wang DI. Effects of substratum morphology on cell physiology. Biotechnol Bioeng. 1994;43(8):764-771.

40. Shin TJ, Park SY, Kim HJ, Lee HJ, Youk JH. Development of 3-D poly(trimethylenecarbonate-co-epsilon-caprolactone)-block-poly (p-dioxanone) scaffold for bone regeneration with high porosity using a wet electrospinning method. Biotechnol Lett. 2010;32(6):877-882.

41. Tong HW, Wang M, Li ZY, Lu WW. Electrospinning, characterization and in vitro biological evaluation of nanocomposite fibers containing carbonated hydroxyapatite nanoparticles. Biomed Mater. 2010; 5(5):054111.

42. Fujihara K, Kotaki M, Ramakrishna S. Guided bone regeneration membrane made of polycaprolactone/calcium carbonate composite nano-fibers. Biomaterials. 2005;26(19):4139-4147.

43. Kulterer B, Friedl G, Jandrositz A, et al. Gene expression profiling of human mesenchymal stem cells derived from bone marrow during expansion and osteoblast differentiation. BMC Genomics. 2007;8:70.

44. Bruder SP, Jaiswal N, Haynesworth SE. Growth kinetics, self-renewal, and the osteogenic potential of purified human mesenchymal stem cells during extensive subcultivation and following cryopreservation. $J$ Cell Biochem. 1997;64(2):278-294.

45. Chun C, Lim HJ, Hong KY, Park KH, Song SC. The use of injectable, thermosensitive poly(organophosphazene)-RGD conjugates for the enhancement of mesenchymal stem cell osteogenic differentiation. Biomaterials. 2009;30(31):6295-6308.

46. Stigbrand T. Present status and future trends of human alkaline phosphatases. Prog Clin Biol Res. 1984;166:3-14.

47. Datta N, Pham QP, Sharma U, Sikavitsas VI, Jansen JA, Mikos AG. In vitro generated extracellular matrix and fluid shear stress synergistically enhance 3D osteoblastic differentiation. Proc Natl Acad Sci U S A. 2006;103(8):2488-2493.

48. Prabhakaran MP, Venugopal J, Ramakrishna S. Electrospun nanostructured scaffolds for bone tissue engineering. Acta Biomater. 2009;5(8): 2884-2893.

49. Seong JM, Kim BC, Park JH, Kwon IK, Mantalaris A, Hwang YS. Stem cells in bone tissue engineering. Biomed Mater. 2010;5(6):062001.

International Journal of Nanomedicine

\section{Publish your work in this journal}

The International Journal of Nanomedicine is an international, peerreviewed journal focusing on the application of nanotechnology in diagnostics, therapeutics, and drug delivery systems throughout the biomedical field. This journal is indexed on PubMed Central,

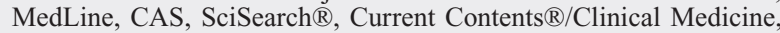

Journal Citation Reports/Science Edition, EMBase, Scopus and the Elsevier Bibliographic databases. The manuscript management system is completely online and includes a very quick and fair peer-review system, which is all easy to use. Visit http://www.dovepress.com/ testimonials.php to read real quotes from published authors. 